\title{
Taking a peek into the editor's office
}

\author{
We are often asked about various aspects of the editor's job, and most frequently about the editorial process \\ after submission. Here, we outline what happens after a manuscript is submitted to the journal and clarify some \\ misconceptions about the editorial process.
}

T he purview of Nature Cell Biology includes cell biology research in the broadest sense, from the fundamental processes that govern cellular homeostasis and responses to external and internal cues, to cellular behaviour in tissues during development and in disease. Given the breadth of the journal's scope, different areas are handled by different editors who stay updated with the current progress in specific fields by engaging with the scientific community. A manuscript submitted to Nature Cell Biology is assigned to the editor responsible for that particular area, who assesses it in detail and discusses it in the editorial team. The handling editor then communicates the decision to the authors. We consider various factors when deciding whether to send a paper out for review, among which is the advance provided over the literature, whether the topic is of broad interest to cell biologists, and the level of development of the dataset. Although we aim for consistent decisions among conceptually related areas, each paper is ultimately evaluated on its own merits.

Selecting reviewers is a crucial part of an editor's work. We aim to engage researchers who are not only experts on the topic but who are also constructive, fair and timely in their assessment (see also, Nat. Cell Biol. 19, 1005; 2017). Although we welcome referee suggestions by our authors, we are not bound to follow them. However, we do honour up to three referee exclusions if authors ask us to avoid certain experts. Peer review at Nature Cell Biology is anonymous unless a reviewer chooses to reveal their identity and, since 2015, we have offered the option of double-blind peer-review for authors who wish to remain anonymous to reviewers (discussed here, Nat. Cell Biol. 17, 195; 2015).

Once the referees submit their reports, the handling editor discusses these in detail in the team, including with the chief editor, to decide whether to pursue the manuscript further. Papers submitted to our journal may span several areas and techniques, meaning that referee requests may be quite diverse and far-reaching in scope, illustrating the different scientific interests within the referee pool. When deciding whether to proceed further with a manuscript,
Nature Cell Biology editors do not simply consider the number of positive versus negative referee recommendations. Rather, we critically evaluate the perspective of each referee and of the authors, focusing on the paper's key messages and whether or how they can be strengthened, in order to reach a fair and informed decision. When inviting a revision, apart from sending authors the full referee reports, we also provide detailed guidance regarding the necessary revisions by synthesizing the referee points that the authors should address as a priority, consolidating similar concerns raised by different reviewers. In addition we circumscribe the scope of the revisions by editorially overruling referee requests that are peripheral or too open-ended. The referees are informed of the decision and the comments of the other referees, including points on which we overruled, so that they are aware of the rationale behind our decision. Reaching the post-review decision is an active process during which the editor may engage in further discussions with all parties involved, for example by asking referees for clarifications or comments on issues raised by another referee, or by consulting with the authors about specific referee questions.

Agreeing to review a paper for Nature Cell Biology is taken as agreeing to assess subsequent versions, as we strive to maintain consistency in the reviewing process. However, in cases of contrasting views by referees or key disagreements between authors and referees, we enlist independent experts to provide input on those specific points (see also Nat. Cell Biol. 19, 1005; 2017).

Manuscripts may not be successful after peer-review for various reasons, including limited novelty, interest to a more specialised section of the field, questionable biological relevance, insufficient mechanistic development, or major technical or conceptual problems raised by the referees. We support the rapid dissemination of research and therefore, we would not invite a revision if addressing key issues would likely require more than six months.

When authors contact the journal because they disagree with a negative decision, we consider their scientific arguments, proposals for additional experiments and, if the paper was peer reviewed, their responses to the referee concerns, in detail. However, as we base our decisions on careful editorial assessments and discussions, we are unlikely to reverse a decision unless an important aspect was missed by editors or referees, or substantial revisions have addressed our previous concerns.

We appreciate that submitting a manuscript to peer-review and revision involves precious time and effort by authors and referees so we strive to provide an efficient editorial process and timely, well-considered decisions. To ensure that publication is not unduly delayed we also consider whether a manuscript we decline would be suitable for another journal in the Nature family and whether it might be in the authors' interests to transfer the paper to that journal using our automated manuscript transfer system. Transferring a manuscript rather than submitting afresh may save authors time as it obviates the need to reformat files. For peer-reviewed manuscripts, referee reports and identities are also transferred, enabling the receiving journal to make a decision based on the peer-review process at the originating journal without having to initiate a fresh round of review. If authors have granted their permission and we consider a paper particularly suitable for one of our sister journals, we may consult with our colleagues at that journal on whether they would pursue the paper further (and for peerreviewed manuscripts, what revisions they would expect), thereby providing authors with a potential route to rapid publication elsewhere. More on manuscript transfers can be found at https://go.nature.com/2Mkc1N2.

Nature Cell Biology aims to publish the most striking and high quality cell biology research while offering a constructive and timely editorial process and peer-review. To that end, we engage closely with our authors and referees and welcome suggestions on how to improve aspects of the process at cellbio@nature.com.

Published online: 26 September 2018 https://doi.org/10.1038/s41556-018-0212-2 